\title{
ESTIMATION OF MOMENTS ACTING ON THE PELVIS IN STANDING AND IN TWO DIFFERENT SITTING POSTURES
}

\section{Bálint Héra, József Zalavári}

Budapest University of Technology and Economics, Department of Machine and Product Design hera.balint@gt3.bme.hu

\begin{abstract}
According to popular consideration prolonged sitting posture in work can cause pain at different regions of the back. Many researches aiming to define the relationship between these two, and as a result of noticing the problem many design solutions can be found, some of which are considered as alternative. Healthy sitting posture should be mean that with anterior rotation of the pelvis the curve of the lumbar spine would be neutral or more preferably lordotic. In sitting the muscle balance changes thus there is more muscle activity needed for erection of the trunk and anterior rotation of the pelvis. In this study muscle activity in standing and in two different sitting postures is estimated as a difference of the two moments acting on the pelvis, which are the upper body weight moment and the passive elastic moment. Further investigation of the effects of hip-flexing thigh muscles or other passive tissues on pelvic range of motion is warranted.
\end{abstract}

Keywords: sitting biomechanics, standing, passive moment, active moment, weight moment, hip joint angle

\section{How sedentary work style affects health}

\subsection{Sitting at work}

Sitting posture is more preferably during activities in that changing the place is not necessary. Under constant loading, those thigh and trunk muscles that keeping the upper body in an erect posture can be exhausted. Sitting on a chair the upper body weight is supported by the seat so that many of the muscles are relaxed. Most of the craftsmen were on the spot, just like today's office workers and computer workers. Driving car, watching TV and using PC we spent additional hours sitting after official work time.

The harmful effects of a sedentary work style were first described around 1700 by the Italian physician Bernardino Ramazzini who considerably grounded occupational medicine.
"Those who sit at their work and are therefore called 'chairworkers,' such as cobblers and tailors ... become bent, humpbacked, and hold their heads down like people looking for something on the ground; this is the effect of their sedentary life (...)". ${ }^{1}$ Besides craftsmen he also mentioned clerks. Ramazzini was the first who established that the constant sitting posture is among risk factors of diseases, and he noticed all chairworkers are suffering lumbago. The classic word lumbago refers to a sudden pain arising around the lower back and the sacrum, which warns to the overload or functional mismatch of the lower spine. ${ }^{2}$ There are many today's examples for occupational sitting, such as occupational driving or different office jobs. The latter is spreading, engineers, physicians and managers spending hours in front of the computer. Growing attention is paid to the back pain and body postures of school children. 


\subsection{Consequences of prolonged sitting posture}

According to popular consideration prolonged sitting posture in work can cause pain at different regions of the back. In my research possible evading of low back pain is examined. Low back pain (LPB) is the type of back pain arising at the sacrum and lumbar vertebras. Causes of mechanical low back pain are important. These are for example the non-specific injuries of the musculoskeletal system; herniated discs; compressing of nerve roots; degenerative changes in the intervertebral disc or joint; fracture of the vertebrae; injury of muscles and ligaments; etc. Low back pain is a serious health issue in the developed countries with over half of sedentary workers reporting LBP during their lifetime. ${ }^{3}$

There is a debate among researchers about the relationship between sitting posture and pain sensed in the back. Some studies demonstrated associations between a history of sedentary work, low-back pain prevalence and degenerative changes of the intervertebral disc. ${ }^{4,5}$ However, other studies do not found such relationship. ${ }^{6,7}$ In their review of studies which are examining the connection between occupational sitting and low back pain Lis and colleagues ${ }^{8}$ found that sitting by itself does not increase the risk of LBP. However, sitting for more than half a workday in combination with awkward posture does increase the likelihood of having LBP. They also found that awkward posture itself is a risk factor. Opposite to these results, duration of prolonged sitting was considered important by many researchers. Spyropoulos and colleagues ${ }^{9}$ studied the prevalence of back pain among Greek public office workers. They have revealed that among significant determinants for predicting LBP occurrence are clerk body position while sitting and sitting time of greater than 6 hours. Janwantanakul and colleagues ${ }^{10}$ developed a risk score to iden- tify office workers likely to have LBP. During data collection for the score called The BROW (The Back pain Risk score for Office Workers), respondents were asked about whether they continuously sat or stood for more than two hours a day. Studying Iranian office workers, Razee and colleagues ${ }^{11}$ conclude that sitting work style more than four hours had a positive association with increased likelihood of occurrence of $L B P$.

The great prevalence of back pain in office workers could indicate relationship between sedentary work style and LBP. In the study of Razee and colleagues lifetime prevalence of back pain was $91.2 \%$, and this result for last twelve month was $37,3 \%$. Considering the preceding 12 month of their study, Griffiths and colleagues $^{12}$ reporting a $70 \%$ lifetime prevalence of LBP for people who spent working with computer more than eight hours a day.

\section{Sitting posture and pain}

How the musculoskeletal system changes when sitting down, and what is the relationship between these changes and pain sensation at the lower back? Answering this we have to examine human body at the level of its anatomical units. One function of the pain is to call attention to the possibility of an injury. In case of the musculoskeletal system the muscles and ligaments are those that can generate pain via nerve roots. When sitting down, the rotation of specific bones related to each other (Figure 1) effects two important changes. First, the arrangement of the lumbar spine changes which is followed by the changes of the loading properties of the soft tissues connecting to it and, in extreme conditions, injury. Second, the muscle equilibrium changes, therefore restoration of the neutral lumbar spine shape needs more muscle activity, which is followed by the changes of the loading properties of the mus- 


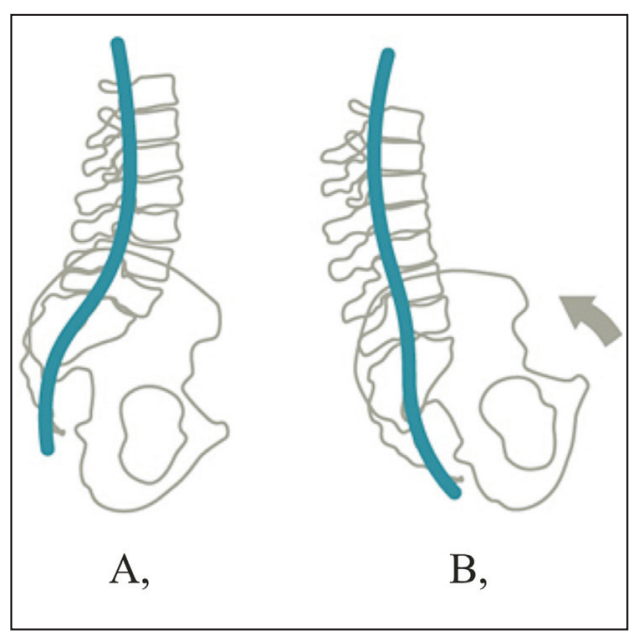

Figure 1. Pelvic position and curvature of the lumbar spine in standing A,) and in sitting with a hip joint angle of $90^{\circ} \mathrm{B}$,) (Figure is adapted from Stumpf and Walker ${ }^{13}$ )

cles, too. Summing up what has been said, pain can frequently arise in soft tissues or in the muscles erecting the trunk or flexing the hip. These muscles are some of the back muscles erecting the trunk (erector spinae, multifidus lumborum), the quadratus lumborum muscle and the iliopsoas muscle which flexing the hip.

With RTG traces Keegan ${ }^{14}$ represented posterior rotation of the pelvis in sitting compared with standing which is followed by flattening or dependent on the posture even forward flexing (kyphosis) of the lumber curve. Keegan considered kyphosis as harmful for the lumbar intervertebral disc, however, others reported the beneficial influence of lumbar kyphosis. Harrison and colleagues ${ }^{15}$ noticed that in kyphotic position of the lumbar spine a greater amount of intradiscal pressure can be experienced that can cause degeneration of the lumbar intervertebral disc. In kyphosis the shear force pulls the disc posterior possibly leading to the bulging of the disc which can contact the sensitive ligaments and nerve roots by generating pain.

\section{Passive moment acting on the pelvis}

There are three main moment acting on the pelvis: active moment, passive moment, and the moment comes from the upper body weight. The active moment is caused by the conscious muscle activity for conscious moving or counterbalancing a joint. The passive moment comprises the muscle passive resistance against the rotation of a joint, and the resistance of other soft tissues such as tendons. ${ }^{16}$ The pelvis supports the weight of the upper body portion transmitted by through the spine at the sacroiliac joint. The acting line of the body weight does not intersect the hip joint rotation center therefore it causes a moment on the pelvis.

Riener and Edrich ${ }^{17}$ examined the resistance of lower extremities against moment which they considered as the passive elastic moment of the hip joint which can be seen as equivalent to the passive moment acting on the pelvis. In their experimental study (Figure 2) they have the subject lying supported with a horizontal surface under the back so the legs can be moved. They lifted one segment of a joint of the limb through a dynamometer while the considered joint angle was measured with an electro-gorniometer and other joints were fixed. Total moment of the joint was calculated from the force $F$ measured and the $l$ distance of the cell from the estimated joint center of rotation. This moment consists of a passive elastic and a passive dissipative moment, as well as a dynamic moment caused by inertial effects, and a moment of the gravity acting on the legs. ${ }^{17}$ The dissipative moment is caused by the viscoelastic properties of muscles. The limb was moved very slowly (quasi-statically) so that any dynamic effects are negligible. The gravitational moment was calculated from the mass $m$ and the center of gravity $l_{C O G}$ of limbs and orthosis distal to the joint investigated. According to these considerations, the passive 


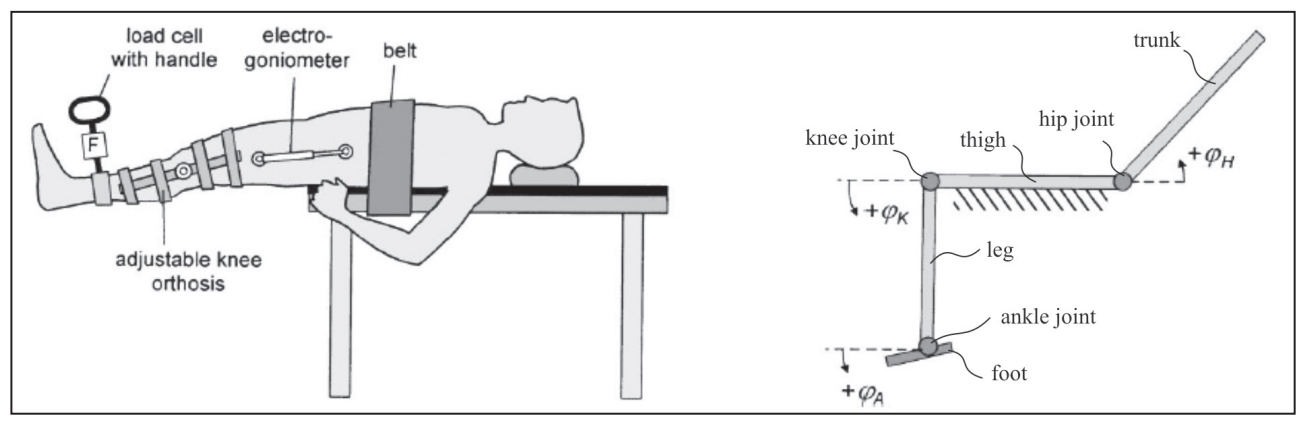

Figure 2. Experimental setup for measuring the passive hip joint moment versus joint angle.

The adjacent joint angles were fixed with an adjustable, knee orthosis, or a backrest. The goniometer for measuring joint angles is comprised of two potentiometers connected by a telescopic arm (Murray, 1992). Geometric definitions are illustrated in the right (Figure adapted from Riener and Edrich with permission)

(elastic and dissipative) moment of a joint can be calculated as:

$M_{\mathrm{pas}} \approx F l-m g l_{\mathrm{COG}} \sin \left(\varphi-\varphi_{\mathrm{r}}\right)$,

where $g$ is the amount of gravity, $\varphi$ is the joint angle and $\varphi_{r}$ is the resting angle, where legs are hanging vertical.

From the measurements of ten subjects using the least square method they defined three so called double exponential function which de- scribe the passive elastic joint moment of the hip, knee and ankle of a generic subject. One of their function with which passive elastic moment of the hip can be calculated ${ }^{17}$ is:

$$
\begin{aligned}
& M_{\mathrm{H}}=\exp \left(1.4655-0.0034 \varphi_{\mathrm{K}}-0.0750 \varphi_{\mathrm{H}}\right)-\exp \\
& \left(1.3403-0.0226 \varphi_{\mathrm{K}}+0.0305 \varphi_{\mathrm{H}}\right)+8.072
\end{aligned}
$$

Where $\varphi_{\mathrm{K}}$ is the knee joint angle and $\varphi_{\mathrm{H}}$ is the hip joint angle (Figure 2) both in degrees. In Figure 3 hip joint moment in three different knee angle arrangement is shown.

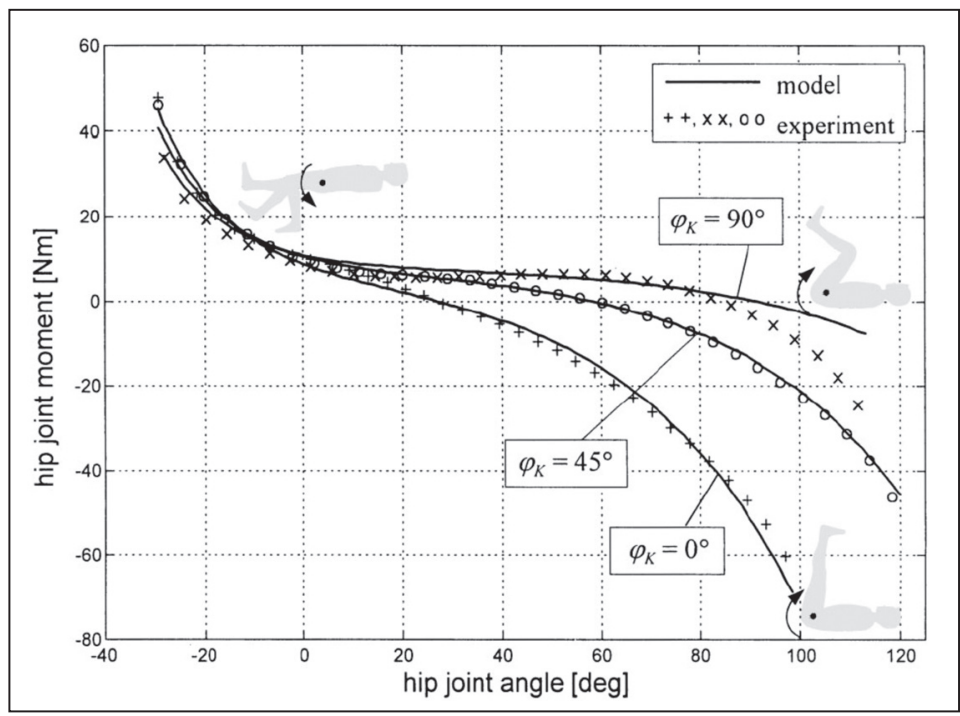

Figure 3. Measured hip joint moment versus hip angle in different knee positions (adapted from Riener and Edrich with permission) 


\section{Moments calculated in this study}

In standing both knee and hip joint angle are $0^{\circ}$ while in the popular perpendicular sitting position both are about $90^{\circ}$. Besides these postures there is another sitting posture we can often meet which is considered as alternative. To these three positions passive elastic joint moment and upper body weight moment acting on the pelvis was calculated and as a different of these two active muscle moments was estimated.

\subsection{The passive elastic moment of the hip joint}

The passive moment calculated with the (2) double exponential function given by Riener and Edrich are shown in Table 1. The positive amount of the moment in case of each posture means that the passive moment has a sagittally forward rotating effect on the pelvis. This moment together with the active muscle moment compensate the moment of the upper body weight. With an increase of the hip joint angle there is an increase in the passive moment and an increase active moment is needed to compensate body weight moment. In case of hip joint angle of $90^{\circ}$ the passive moment is around zero thus the pelvis and the upper body are stabilized with active muscles.

\begin{tabular}{|c|c|c|c|c|c|c|}
\cline { 2 - 7 } \multicolumn{1}{c|}{} & \multicolumn{2}{c|}{1.} & \multicolumn{2}{c|}{2.} & \multicolumn{2}{c|}{3.} \\
\cline { 2 - 7 } \multicolumn{1}{c|}{} & hip & knee & hip & knee & hip & knee \\
\hline $\begin{array}{c}\text { angle } \\
{\left[{ }^{\circ}\right]}\end{array}$ & 0 & 0 & 45 & 45 & 90 & 90 \\
\hline $\begin{array}{c}\mathbf{M}_{\text {pas }} \\
{[\mathrm{Nm}]}\end{array}$ & \multicolumn{2}{|c|}{8.6} & \multicolumn{2}{c|}{2.7} & \multicolumn{2}{c|}{0.3} \\
\hline
\end{tabular}

Table 1. Passive elastic moment acting on the pelvis (hip join) in three different body postures

\subsection{Upper body weight moment}

The load of the upper body weight was substituted with a vertical force acting at the center of the sacroiliac joint. This force can be sub- stituted with the L5-S1 joint force given in the study of Arjmand and colleagues ${ }^{18}$ who published data calculated with two different model. The single-level optimization-assisted EMGdriven (EMGAO) model contains a process in which the three different components of the joint are estimated with the optimization of preliminary estimated EMG-driven trunk muscle force. The multi-level kinematics-driven (KD) model is a finite element model for a specific part of the musculoskeletal system which contains rigid solid beam elements providing the cumulated nonlinear stiffness and load-displacement relationship of the joint segments.

There is a difference in magnitude of moment arms in case of the three mentioned body posture because the rotation axis of the pelvis changes. In standing position the axis of rotation of the pelvis intersects the center of rotation of the two hip joints while in sitting this axis is defined by the connecting points of the ischial tuberosities and the seat surface. The magnitude of moment arm is given by the length of the transverse section which connects the acting line of the upper body weight to the rotation axis of the pelvis.

The angle of pelvic tilt (PT) for standing and $90^{\circ}$ sitting was given by Endo and colleagues. ${ }^{19}$ For the calculation of $\mathrm{PT}$ in the $45^{\circ}$ sitting posture it was estimated that the magnitude of pelvis rotation is half of the magnitude of pelvic rotation between standing and $90^{\circ}$ sitting posture. For the calculation of a vectors between two anatomical points of the pelvis and their lengths and their angle in relation to each other (Figures 4-7), coordinates data were given from the study of Reynolds and colleagues ${ }^{20}$ who measured the pelvis in a tilt angle related to the natural standing body posture and divided the measurements into three anthropometric group (small female, medium male and large male). 
The upper body weight moments related to the three postures are:

$\mathrm{M}_{\mathrm{st}}=\mathrm{F}_{\mathrm{i}}^{*} \mathrm{k}_{\mathrm{st}}$

$\mathrm{M}_{\mathrm{si}, 45^{\circ}}=\mathrm{F}_{\mathrm{i}}^{*} \mathrm{k}_{\mathrm{si}, 45^{\circ}}$

$\mathrm{M}_{\mathrm{si}, 90^{\circ}}=\mathrm{F}_{\mathrm{i}}{ }^{*} \mathrm{k}_{\mathrm{si}, 90^{\circ}}$

where $\mathrm{M}_{\mathrm{st}}$ and $\mathrm{k}_{\mathrm{st}}$ are upper body weight moment and the moment arm in standing position, respectively; $\mathrm{M}_{\mathrm{si}, 45^{\circ}}$ and $\mathrm{k}_{\mathrm{si}, 45^{\circ}}$ are upper body weight moment and moment arm in $45^{\circ}$ sitting position, respectively; and $\mathrm{M}_{\mathrm{si}, 90^{\circ}}$ and $\mathrm{k}_{\mathrm{si}, 90^{\circ}}$ are upper body weight moment and moment arm in $90^{\circ}$ sitting position, respectively. The index $i$ relates to the model type $\mathrm{KD}$ or EMGAO that have been used for calculating the force. In function 3, 4, and 5 each $k$ is the lever arm, where

$\mathrm{k}_{\mathrm{st}}=\sin \varphi_{\mathrm{st}}{ }^{*} \underline{\mathrm{d}}_{\mathrm{st}} \mid$

$\mathrm{k}_{\mathrm{si}, 45^{\circ}}=\sin \varphi_{\mathrm{si}, 45^{\circ}} * \underline{\mathrm{d}}_{\mathrm{si}, 45^{\circ}} \mid$

$\mathrm{k}_{\mathrm{si}, 90^{\circ}}=\sin \varphi_{\mathrm{si}, 90^{\circ}}{ }^{*} \underline{\mathrm{d}}_{\mathrm{si}, 90^{\circ}} \mid$

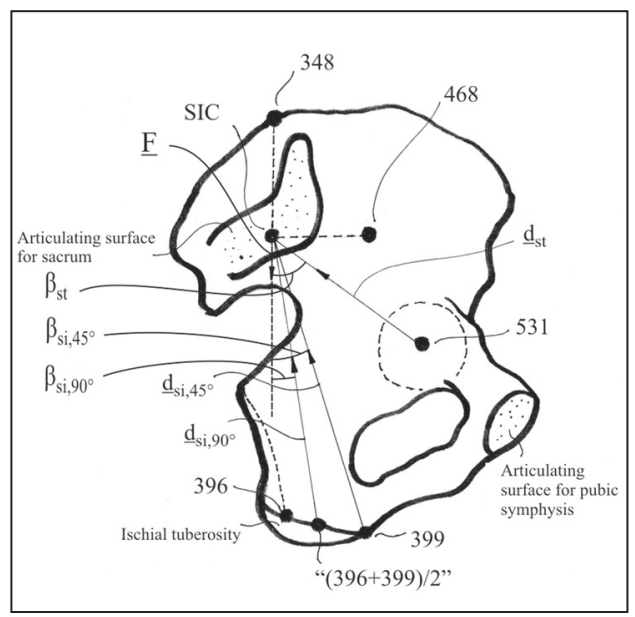

Figure 4. Anatomical points and vectors on the lateral view of the pelvis which have been used for calculating upper body weight moment. 531 is the center of hip joint; SIC is the sacroiliac joint center; 348 and 468 are two points which define SIC. The code numbers 348, 396, 399, 468, 531 are matched with anatomical points due to identification by Reynolds and colleagues ${ }^{20}$

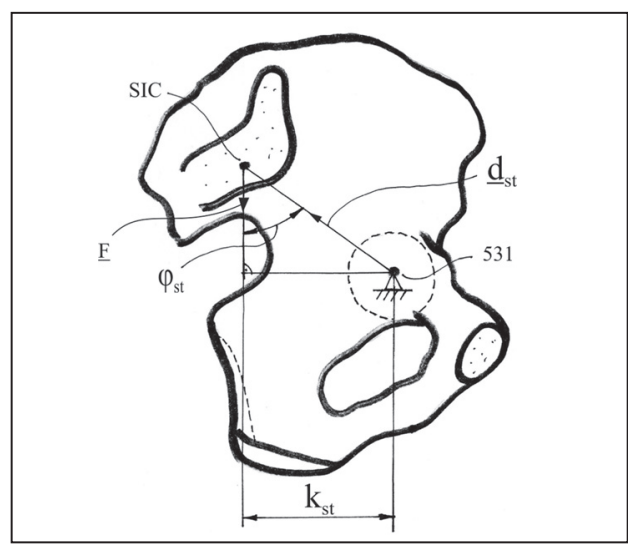

Figure 5. The lever arm of the upper body weight moment in standing posture

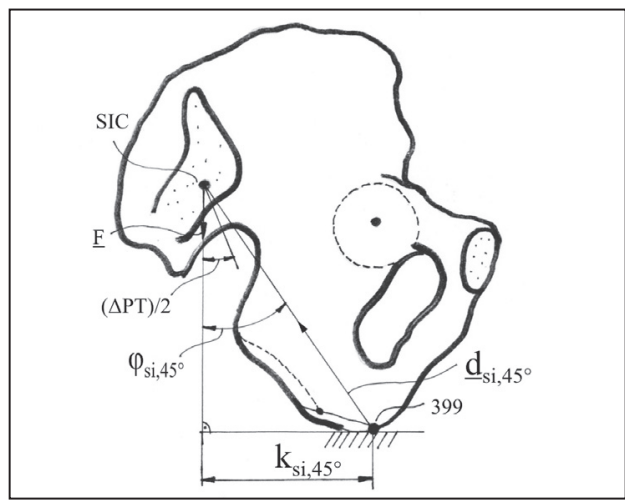

Figure 6. The lever arm of the moment in sitting with hip joint angle of $45^{\circ}$

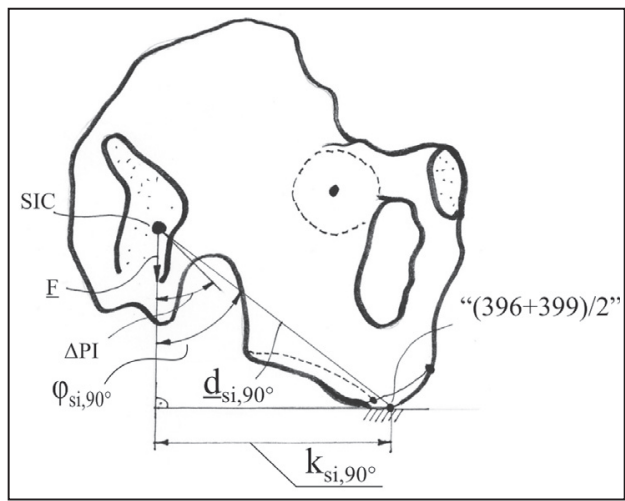

Figure 7. The lever arm of the moment in sitting with hip joint angle of $90^{\circ}$ 
where $\underline{\mathrm{d}}_{\mathrm{st}}, \underline{\mathrm{d}}_{\mathrm{si}, 45^{\circ}}$ and $\underline{\mathrm{d}}_{\mathrm{si}, 90}$ are vectors which connect the acting line of the upper body weight and the rotation axis of the pelvis, and are transverse to each of those; and $\varphi_{\mathrm{st}}, \varphi_{\mathrm{si}}, 45^{\circ}$ and $\varphi_{\text {si } 90^{\circ}}$ are angles which are sum of PT and the smaller angle defined by vector $\underline{\mathrm{d}}$ and upper body weight line. Results are shown in Table 2.

\begin{tabular}{|c|c|c|c|}
\hline & \multirow{2}{*}{$\begin{array}{c}\text { body } \\
\text { size/gender }\end{array}$} & \multicolumn{2}{|c|}{ model type } \\
\hline & & $\mathrm{KD}$ & EMGAO \\
\hline \multirow{3}{*}{$\mathbf{M}_{\mathrm{st}}[\mathrm{Nm}]$} & small female & 9.8 & 10.7 \\
\hline & medium male & 12.5 & 13.7 \\
\hline & large male & 12.4 & 13.6 \\
\hline \multirow{3}{*}{$\mathbf{M}_{\mathrm{si}, 45^{\circ}[\mathrm{Nm}]}$} & small female & 18.8 & 20.7 \\
\hline & medium male & 23.3 & 25.7 \\
\hline & large male & 24.3 & 26.9 \\
\hline \multirow{3}{*}{$\mathbf{M}_{\mathrm{si}, 90^{\circ}}[\mathrm{Nm}]$} & small female & 29.5 & 32.8 \\
\hline & medium male & 32.0 & 35.6 \\
\hline & large male & 31.5 & 35.0 \\
\hline
\end{tabular}

Table 2. Upper body weight moment acting on the pelvis (hip joint) in three different body postures

\section{Conclusion}

\subsection{Estimating active muscle moment of the pelvis}

Comparing the calculated results of passive and upper body weight moments of the pelvis it is found that the order of magnitude of passive moment is only comparable with the body weight moments in standing position. In both sitting posture with backward rotation of the pelvis there is an increase in body weights moment and a decrease in passive moment. Due to higher muscle activity exhausting of muscles and adaptation to a slumped position could be experienced after shorter period of sitting. Load on ligaments and discs and other muscles will be increased which can be harmful. In Table 3 calculated moments and active muscle moments estimated as a difference between those moments are shown. The Table 3 layout is simplified by averaging the upper body weight moment based on loading forces calculated with the two different models (KD, EMGAO) by Arjmand and colleagues.

On the basis of results of active muscle moment (Table 3) it is found that due to the lower muscle activity needed for it the $45^{\circ}$ sitting posture can be maintained longer.

\subsection{Limit of Study}

Results have to be considered for a generic subject. Differences in moments of a specific subject could be due to the use of a generic function of passive elastic hip joint moment, a generic L5-S1 joint force, and generic lever arms of upper body weight. There is noticeable gap between measured and with model calculated data of passive elastic hip joint moment given by Riener and Edrich at the end of the range of motion of the joint.

\begin{tabular}{|c|c|c|c|c|c|c|c|c|c|}
\hline & \multicolumn{9}{|c|}{ hip joint moment } \\
\hline & \multicolumn{3}{|c|}{ Body weight moment $[\mathrm{Nm}]$} & \multicolumn{3}{|c|}{ Passive elastic moment $[\mathrm{Nm}]$} & \multicolumn{3}{|c|}{ Active muscle moment $[\mathrm{Nm}]$} \\
\hline & st & $\mathrm{si}, 45^{\circ}$ & si, $90^{\circ}$ & st & $\mathrm{si}, 45^{\circ}$ & $\operatorname{si}, 90^{\circ}$ & st & $\mathrm{si}, 45^{\circ}$ & si, $90^{\circ}$ \\
\hline small female & 10.2 & 19.7 & 31.2 & \multirow{3}{*}{8.6} & \multirow{3}{*}{2.7} & \multirow{3}{*}{0.3} & 1.6 & 17.0 & 30.9 \\
\hline medium male & 13.1 & 24.5 & 33.8 & & & & 4.5 & 21.7 & 33.5 \\
\hline large male & 13.0 & 25.6 & 33.3 & & & & 4.4 & 22.8 & 33.0 \\
\hline
\end{tabular}

Table 3. Passive elastic moment; upper body weight moment; and active muscle moment estimated as a different of these two moments 


\section{REFERENCES}

1. Franco $G$, Franco $F$. Voices from the past-De Morbis Artificum Diatriba (Diseases of Workers). American Journal of Public Health 2001; 91(9):1380-2.

2. Lumbágó. [cited 2014 Jan 05]. Available from: URL: www.wikipedia.hu/lumbago.

3. Lloyd M, Gauld S, Soutar C. Epidemiologic study of back pain in miners and office workers. Spine 1986;11:136-40.

4. Ewans $W$, Jobe $W$, Seibert $C$. A cross-sectional prevalence study of lumbar disc degeneration in a working population. Spine 1999;14:60-4.

5. Videman T, Nurminen $M$, Troup JDG. Lumbar spinal pathology in cadaveric material in relation to history of back pain, occupation, and physical loading. Spine 1990;15:728-40.

6. Bakker E, Verhagen A, van Trijffel E, Lucas C, Koes B. Spinal mechanical load as a risk factor for low back pain: a systematic review of prospective cohort studies. Spine 2009;34:E281-93.

7. Roffey D, Wai E, Bishop P, Kwon B, Dagenais $S$. Causal assessment of occupational sitting and low back pain: results of a systematic review." The Spine Journal 2010;10:252-61.

8. Lis A., Black K, Korn H, Nordin M. Association between sitting and occupational LBP. European Spine Journal 2007;16:283-98.

9. Spyropoulos P, Papathanasiou G, Georgoudis $G$, Chronopoulos E, Koutis H, Koumoutsou F. Prevalence of low back pain in greek public office workers. Pain Physician 2007;10:651-9.

10. Janwantanakul P, Pensri P, Moolkay P, Jiamjarasrangsi $W$. Development of a risk score for low back pain in office workers- a cross-sectional study. BMC Musculoskeletal Disorders 2011; 12:23.
11. Rezaee M, Ghasemi M, Jafari NJ, Izadi M. Low back pain and related factors among iranian office workers. International Journal of Occupational Hygiene 2011;3:23-8.

12. Griffiths KL, Mackey MG, Adamson BJ, Pepper $K L$. Prevalence and risk factors for musculoskeletal symptoms with computer based work across occupations. Work 2012;42:533-41.

13. Stumpf B, Walker BM. The Benefits of Pelvic Stabilization. Zeeland (Michigan): 2003. Sponsored by Herman Miller, Inc.

14. Keegan JJ. Alterations of the lumbar curve related to posture and seating, J Bone and Joint Surg (B) 1953;35:589-603.

15. Harrison DD, Harrison SO, Arthur CC, Harrison DE, Troyanovich SJ. Sitting Biomechanics Part I: Review of the Literature. Journal of Manipulative and Physiological Therapeutics 2000;23:37-47.

16. Johns RJ, Wright V. Relative importance of various tissues in joint stiffness. Journal of Applied Physiology 1962;17:824-8.

17. Riener R, Edrich $T$. Identification of passive elastic joint moments in the lower extremities. Journal of Biomechanics 1999;32:539-44.

18. Arjmand N, Gagnon D, Plamondon A, ShiraziAdl A, Larivičre C. Comparison of trunk muscle forces and spinal loads estimated by two biomechanical models. Clinical Biomechanics 2009; 24:533-41.

19. Endo K. Suzuki H, Nishimura H, Tanaka H, Shishido T, Yamamoto K. Sagittal lumbar and pelvic alignment in the standing and sitting positions. J Orthop Sci 2012;17:682-686. 
20. Reynolds HM, Snow CC, Young JW. Spatial Geometry of the Human Pelvis. Washington DC: Federal Aviation Administration (US), Human Aeromedical Office; 1982 March. Report No.:
FAA-A2-82-9. Sponsored by Washington DC: Federal Aviaton Administration (US), Office of Aviation Medicine; Washington DC: National Highway Traffic Safety Administration.

Authors would like to thank the professional advices to Rita Kiss, director of the BME Biomechanical Cooperative Research Center.

\section{Bálint Héra}

Danubia Szabadalmi és Jogi Iroda Kft.

H-1368 Budapest, Pf. 198

Tel.: (+36) 1 411-8847 